\title{
Correlations among Apparent Diffusion Coefficient and Permeability Parameters from Dynamic Contrast-enhanced MR in Brain Tumor Parenchyma and Peritumoral Area
}

Jing Zhao, Yu Feng, Shao-fu He, Zhi-yun Yang and Jian-ping Chu*

Department of Radiology, The First Affiliated Hospital of Sun Yat-Sen University, China

\begin{abstract}
Purpose: To evaluate the correlations among permeability parameters and Apparent Diffusion Coefficient (ADC) in different brain tumors and different tumor areas.

Materials and methods: Prospectively included 45 patients (M: 23, mean age: 46 y) who were examined by conventional, Diffusion Weighted Image (DWI) and Dynamic Contrast-Enhanced MRI (DCE-MRI). ADC and permeability parameters (volume transfer constant: Ktrans, extra-vascular extra-cellular volume fraction: Ve, reverse volume transfer constant: Kep and initial area under the time-concentration curve: iAUC) were calculated. 6-10 and 4-5 Regions of Interest (ROIs) were manually placed on Tumor Parenchyma (TP) and Peri Tumoral (PT) area, respectively.
\end{abstract}

Results: Irrespective of brain tumor types, mean ADC, in TP and PT, were significantly inversely correlated with mean Ktrans, Ve, iAUC $(P<0.00)$ and positively correlated with mean Kep $(P<0.00)$. Further, specific to gliomas, similar correlations were found in TP and PT area (except mean Kep does not show any correlations with mean ADC in PT); to metastases, only mean Ve in TP area was associated with ADC $(P=0.04)$. Moreover, among the permeability parameters, of TP and PT, Ktrans, Ve and iAUC were mutually positively correlated with each other $(\mathrm{P} \leq 0.03)$.

Conclusion: Intricate correlations were found among permeability parameters and ADC, irrespective of the brain tumor types and ROI positions. Ktrans, Ve and iAUC may have a comparable diagnostic ability to classify brain tumors.

Keywords: Magnetic Resonance Imaging (MRI); Diffusion; Perfusion; Brain neoplasms; Association

\section{Introduction}

Brain tumors are a diverse group of neoplasms and the second leading cause of cancer-related deaths in male ages 20-39 (years of age) [1]. Each type of brain tumor has their own unique tumor microenvironment and is characterized by varying degrees of hypercellularity, nuclear pleomorphism, endothelial proliferation and microvascular density. Nearly all brain tumors are accompanied with the Peri Tumoral (PT) area abnormality, which manifest as high signal intensity on T2-weighted MR imaging. From a pathological point of view, PT area signal abnormality represents a tumor induced interstitial edema. In High-Grade Gliomas (HGG) and Primary Central Nervous System Lymphoma (PCNSL), the PT area signal abnormality is not only caused by the altered interstitial water but also by the infiltration of the scattered tumor cells [2]. In contrast with the HGG and PCNSL, the infiltrated tumor cell could not be found in the PT area of metastases $[3,4]$. Thus, the micro-environment of each type of brain tumor is different from Tumor Parenchyma (TP) to PT area. The frequently applied techniques are Diffusion Weighted Image (DWI) and Dynamic Contrast-Enhanced MRI (DCE-MRI), they reflect the tumor micro-environment from different angles and both have been used as independent imaging biomarkers for brain tumor diagnosis. The Apparent Diffusion Coefficient (ADC) maps represent the freedom of water molecules to diffuse within tissue and the ADC values negatively correlate with tumor cellularity, which could be used in gliomas grading and tumor differentiation [5-8]. ADC is influenced by a number of factors, such as Extracellular Extravascular Space (EES) volume, spatial configuration, membrane permeability and ADC is predominantly affected by extracellular geometry [9].
Pharmacokinetic modeling of DCE-MR imaging data allows estimation of many permeability parameters that affect the delivery and local distribution of the contrast molecules. Ktrans $\left(\mathrm{min}^{-1}\right)$, volume transfer constant, is one of the most important measures of tumor vascular leakiness and has been proved to positively correlate with the gliomas grades [10-12]; Ve, extra-vascular extra-cellular volume fraction, is a direct estimate of the EES volume and it has been considered as a metric of tumor necrosis and inversely correlated with tumor cellularity [13]; Kep (min-1) is reverse volume transfer constant and few studies have showed that Kep was significantly higher in high grade tumors [14-15]; iAUC, initial area under the time-concentration curve, is a model free parameter [16] and it has been used extensively in diagnostic practice.

Several papers and our previous study have showed that ADC and some of the permeability parameters (Ktrans, Ve, Kep and iAUC) in $\mathrm{TP}$ or PT area could significantly differentiate diverse types of brain tumors [10-12,17-19]. However, compared with ADC, a study showed that permeability parameters demonstrated higher diagnostic value [19]. Thus, we hypothesize that, with different brain tumors and in

*Corresponding author: Jian-ping Chu, Department of Radiology, The First Affiliated Hospital of Sun Yat-Sen University, 58th, The $2^{\text {nd }}$ Zhongshan Road, Guangzhou, Guangdong, China, Tel.: +8620-87755766; Ext: 8474; Fax: +862087615805, E-mail: truechu@hotmail.com

Received August 31, 2018; Accepted September 17, 2018; Published September 22, 2018

Citation: Zhao J, Feng Y, He S, Yang Z, Chu J (2018) Correlations among Apparent Diffusion Coefficient and Permeability Parameters from Dynamic Contrast-enhanced MR in Brain Tumor Parenchyma and Peritumoral Area. J Neurol Neurophysiol 9: 470. doi:10.4172/2155-9562.1000470

Copyright: (c) 2018 Zhao J, et al. This is an open-access article distributed under the terms of the Creative Commons Attribution License, which permits unrestricted use, distribution, and reproduction in any medium, provided the original author and source are credited. 
different tumor regions (TP and $\mathrm{PT}$ ), there should exist intricate relationships among permeability parameters and ADC. Investigating correlations among ADC and permeability parameters would provide not only a unique perspective of the parameters themselves but also in understanding the tumor micro-environment. Few studies have been done here $[13,20-22]$ and the results are controversial. Irrespective of the brain tumor types, a study has found that the mean ADC values were significantly inversely correlated with the mean Ktrans, Ve and iAUC and positively correlated with the mean Kep in TP [20]. Specify to gliomas, in TP, Mills SJ et al. showed correlation among each pair of the permeability parameters [21]. However, no correlations were found between permeability parameters and ADC in Mills SJ et al. [13] and Choi HS et al. [22]. The correlations among permeability parameters and ADC in other types of brain tumor and in PT area of gliomas are still lack.

Therefore, we attempted to evaluate the correlations of permeability parameters and ADC based on various of brain tumors and different tumor regions (TP and $\mathrm{PT}$ ) and tried to understand the correlations and interactions of those parameters.

\section{Material and Methods}

From November of 2012 to September of 2013, 71 consecutive patients with suspected brain tumor without enduring radiotherapy, chemotherapy, steroid treatment and stereotactic biopsy were prospectively evaluated by conventional, DWI and DCE-MR. Patients who qualified the following criteria: a) the diagnosis was confirmed by pathology and meningioma also could be diagnosed by clinicalradiological criteria (the tumor showed typical meningioma signal on MR [23], which was followed up for at least 1 year and the size and signal of the tumor had no obvious change); b) the PT area of each tumor was suitable for Region of Interest (ROI) placing (accordingly, the intraventricular tumors were excluded), were included in the study. Finally, 45 patients (male: 23, female: 22 ; mean age: 46 years; age range: 8 y-72y) were included: nine Low Grade Gliomas (LGG), 15 HGG, ten meningiomas, six PCNSL and five metastatic tumors (Figure 1).

\section{MRI protocol and data post-processing}

\section{Conventional MR}

Brain MRI was performed on all patients using a 3-T MR system
(Magnetom Trio, Siemens Medical Solutions, Erlangen, Germany) and 12 phased-array brain coils. Transversal T2-weighted (TR: $4000 \mathrm{~ms}$, TE: $100 \mathrm{~ms}$, FOV: $230 \times 230 \mathrm{~mm}$; slice thickness: $5 \mathrm{~mm}$; slice gap: $0.5 \mathrm{~mm}$; voxel resolution: $0.7 \mathrm{~mm} \times 0.6 \mathrm{~mm} \times 6.0 \mathrm{~mm}$ ), transversal T1-weighted (TR: $400 \mathrm{~ms}$; TE: $8.9 \mathrm{~ms}$; FOV: $230 \times 230 \mathrm{~mm}$; slice thickness: $5 \mathrm{~mm}$; slice gap: $0.5 \mathrm{~mm}$; voxel resolution: $0.9 \mathrm{~mm} \times 0.7 \mathrm{~mm} \times 6.0 \mathrm{~mm}$ ) and coronal fluid-attenuated inversion recovery (TR/TE: $9000 \mathrm{~ms} / 110 \mathrm{~ms}$; inversion time: $2500 \mathrm{~ms}$; FOV: $260 \times 260 \mathrm{~mm}$; slice thickness: $5 \mathrm{~mm}$; slice gap: $0.5 \mathrm{~mm}$; voxel resolution: $0.9 \mathrm{~mm} \times 0.7 \mathrm{~mm} \times 6.0 \mathrm{~mm}$ ) images were obtained firstly. Post-contrast sagittal 3D T1w (TR: $1880 \mathrm{~ms}$; TE: $2.62 \mathrm{~ms}$; section thickness: $1 \mathrm{~mm}$; FOV: $256 \times 256 \mathrm{~mm}$; voxel resolution: $0.7 \mathrm{~mm} \times 0.7 \mathrm{~mm} \times 0.7 \mathrm{~mm}$ ) was obtained after DCE-MR.

\section{Diffusion MR}

DWI was performed by using an axial echo-planar spin-echo sequence (TR: $8700 \mathrm{~ms}$; TE: $88 \mathrm{~ms}$; slice thickness: $3 \mathrm{~mm}$; FOV: $260 \times$ $260 \mathrm{~mm}$; voxel resolution: $1.2 \mathrm{~mm} \times 1.2 \mathrm{~mm} \times 3.0 \mathrm{~mm}$ ). Diffusion was measured in three $b$ values $\left(0,500\right.$, and $\left.1000 \mathrm{~s} / \mathrm{mm}^{2}\right)$ and each nonzero $b$ value has 3 orthogonal directions. ADC maps were automatically generated.

\section{DCE-MRI}

At the same location line of DWI, volumetric interpolated breathhold examination (T1-VIBE) was applied at two different flip angles $\left(2^{\circ}\right.$ and $\left.15^{\circ}\right)$ to calculate the T1-maps. Below were the parameters (TR/TE: $5.21 \mathrm{~ms} / 1.8 \mathrm{~ms}$; slice thickness: $3 \mathrm{~mm}$; FOV: $210 \times 210$ $\mathrm{mm}$; voxel resolution: $1.5 \mathrm{~mm} \times 1.1 \mathrm{~mm} \times 3.0 \mathrm{~mm}$ ). DCE-MRI was acquired using time resolved angiography with stochastic trajectories (TWIST) sequence, the parameters used were following: TR/TE: 4.94 $\mathrm{ms} / 1.93 \mathrm{~ms}$; 10 slices; flip angle: $12^{\circ}$; slice thickness: $3 \mathrm{~mm}$; for each measurement $3.6 \mathrm{~s}$; FOV: $210 \times 210 \mathrm{~mm} ; 75$ measurements; total scan time: $276 \mathrm{~s}$; voxel resolution: $1.6 \mathrm{~mm} \times 1.1 \mathrm{~mm} \times 3.0 \mathrm{~mm}$; contrast media $(0.1 \mathrm{mmol} / \mathrm{kg}$ body weight of Gd-DTPA, Magnevist, Schering, Berlin, Germany); contrast median injection rate: $3.5 \mathrm{~mL} / \mathrm{s}$ for adults and $3 \mathrm{~mL} / \mathrm{s}$ for children, followed by $20 \mathrm{~mL}$ of $0.9 \%$ saline flush using the same injection speed. Infusion started from the fifth measurement.

\section{Data processing}

All DCE-MRI data were transferred to post-processing workstation. Analysis was done by a commercial software tool (TISSUE 4D; Siemens

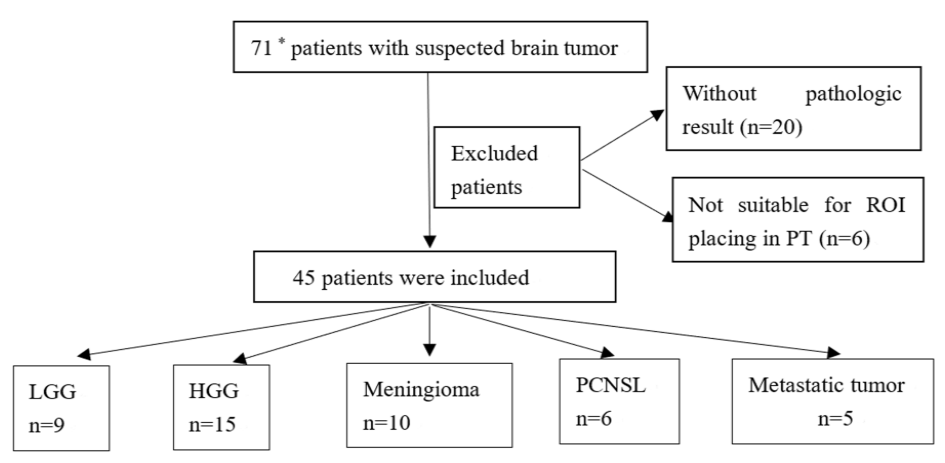

*: All patients without enduring radiotherapy, chemotherapy, steroid treatment and stereotactic biopsy were prospectively evaluated by conventional, DWI and DCE-MR.

PT: Peritumoral area; LGG: low grade glioma; HGG: High grade glioma; PCNSL: Primary central nervous system lymphoma

Figure 1: A flowchart to show how to select patients. 
Healthcare, Erlangen, Germany). A value for the arterial input function was automatically calculated using the software without an arterial ROI. TISSUE $4 \mathrm{D}$ that is based on two compartmental models [2425]. analyze the data in three points of view: 1) three permeability parameters maps (Ktrans, Ve, and Kep) were automatically calculated from the fitted model and the mean values of permeability parameters in each voxel of the drawn ROI were recorded; 2) The corresponding mean value of iAUC in each ROI was recorded either.

\section{ROI placement}

ROI analysis was according to the recommendation of early studies [26-28]. ROI was placed after consensus of two experienced radiologists. The ROIs were placed within TP and PT (within a $1 \mathrm{~cm}$ distance from the outer enhancing tumor margin) area. The size of ROIs was similar to each other $\left(0.25 \mathrm{~cm}^{2}-0.35 \mathrm{~cm}^{2}\right)$. Six-ten (depending on the size of the lesion) and four-five ROIs were separately placed on TP and PT area, according to enhanced T1 weighted images, permeability parameter and ADC maps. Avoiding the necrosis and cystic component, or, if the lesion did not show enhancement, such as LGG, T1 weighted, T2 weighted and FLAIR (fluid attenuated inversion recovery) images were also needed for ROI placement. Total 874 ROIs were placed within TP (permeability parameters maps: 450; ADC map: 424) and 447 ROIs were drawn on PT area (permeability parameters maps: 223; ADC map: 224).

\section{Statistical analyses}

Statistical analysis was carried out using the software (SPSS 16.0 Chicago Illinois USA). Correlations among permeability parameters and $\mathrm{ADC}$ value were calculated by Spearman correlation analysis. Figures of correlation matrices and scatter plots were made by $\mathrm{R}$ package ( $\mathrm{R}$ 3.1.2, Vienna, Austria). The correlation matrices were made by "corrplot" package of R software, and scatter plots were made using plot function of $\mathrm{R}$ software. $\mathrm{P}<0.05$ was considered statistically significant.

\section{Results}

Irrespective of brain tumor types, mean $\mathrm{ADC}$, in $\mathrm{TP}$ or $\mathrm{PT}$, were significantly inversely correlated with the mean value of Ktrans $(\mathrm{rTP}=$ -0.33; $\mathrm{p}<0.00 ; \mathrm{rPT}=-0.32 ; \mathrm{P}<0.00)$, Ve $(\mathrm{rTP}=-0.42 ; \mathrm{P}<0.00 ; \mathrm{rPT}=-0.44$; $\mathrm{P}<0.00)$, iAUC $(\mathrm{rTP}=-0.39 ; \mathrm{P}<0.00 ; \mathrm{rPT}=-0.57 ; \mathrm{P}<0.00)$ and positively correlated with mean Kep $(r \mathrm{TP}=0.42 ; \mathrm{P}<0.00 ; \mathrm{rPT}=0.35 ; \mathrm{P}<0.00)$. Further, Table 1 presented the strong inter-permeability parameter correlations in TP. The correlation matrices showed that the correlation degrees among these different parameters (Figure 2).

Specifically, the correlation between each of the permeability parameter and ADC in different types of brain tumor and ROIs (TP and PT) were recorded in Table 2. Within TP: 1) the similar correlations as Figure 2 were found in LGG and HGG; 2) In PCNSL, the ADC values were significantly inversely correlated with the Ktrans, Ve and iAUC values but no correlation was found with the Kep values; 3) In metastases, only Ve showed inverse correlation with ADC; 4) In meningioma, the $\mathrm{ADC}$ mean values were significantly positively correlated with each of the mean value of Ktrans, Kep and iAUC, but no correlation was presented between Ve and ADC (Figure 3).

For PT area: 1) only iAUC manifested inverse correlation with ADC in LGG; 2) In HGG, ADC values were significantly inversely correlated with the mean values of Ve and iAUC and positively correlated with the Kep values; 3 ) For PCNSL, only Kep showed positive correlation with ADC; 4) For metastases, there were no correlations existed among permeability parameters and ADC; 5) For meningioma, the ADC had inverse correlation with iAUC but positive correlation with Kep. The

\begin{tabular}{|l|l|c|c|c|}
\hline \multirow{2}{*}{$\begin{array}{l}\text { Features being } \\
\text { correlated }\end{array}$} & \multicolumn{2}{|c|}{ TP } & \multicolumn{2}{c|}{ PT } \\
\cline { 2 - 5 } & $\mathbf{r}$ & $\mathbf{1}$ & \multicolumn{1}{c|}{$\mathbf{p}$} \\
\hline $\mathrm{K}^{\text {trans }}$ with Ve & 0.76 & $<0.000^{*}$ & 0.57 & $<0.000^{*}$ \\
\hline $\mathrm{K}^{\text {trans }}$ with iAUC & 0.87 & $<0.000^{*}$ & 0.63 & $<0.000^{*}$ \\
\hline Ve with iAUC & 0.87 & $<0.000^{*}$ & 0.92 & $<0.000^{*}$ \\
\hline Kep with K ${ }^{\text {trans }}$ & -0.15 & $0.001^{*}$ & 0.00 & 0.99 \\
\hline Kep with iAUC & -0.36 & $<0.000^{*}$ & -0.68 & $<0.000^{*}$ \\
\hline Kep with Ve & -0.64 & $<0.000^{*}$ & -0.67 & $<0.000^{*}$ \\
\hline
\end{tabular}

Table 1: The inter-permeability parameters correlations in Tumor Parenchyma (TP) and Peri Tumoral (PT) area, irrespective of brain tumor type; *Significant.

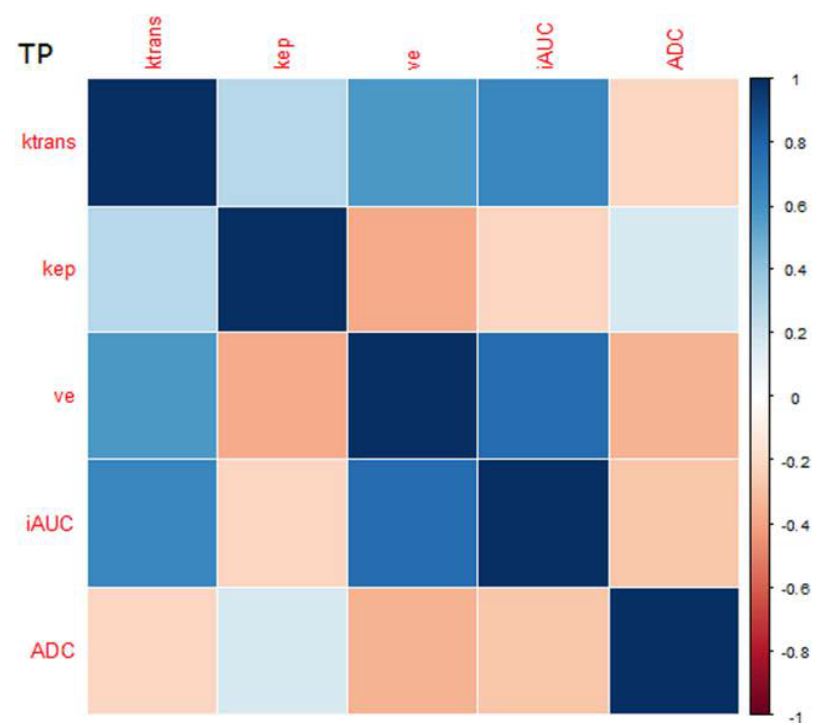

Figure 2: Correlation matrices among permeability parameters (Ktrans, Kep, Ve and iAUC) and ADC, irrespective of brain tumor types; TP: In tumor parenchyma, Ktrans $(r=0.87)$ and $V e(r=0.87)$ showed the equally highest correlation with iAUC; PT: In peritumoral area, Ve and iAUC showed the highest association ( $r=0.92)$.

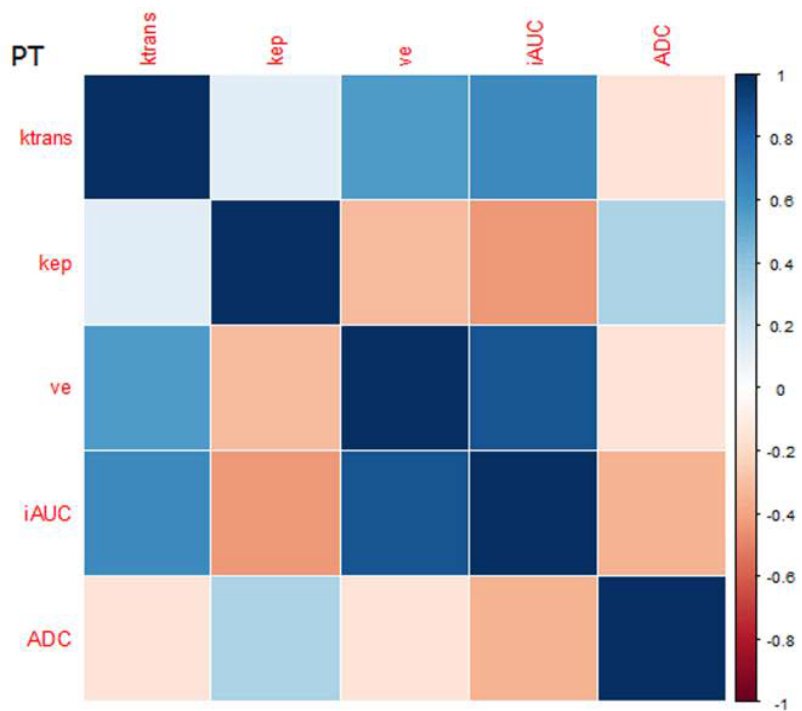


Citation: Zhao J, Feng Y, He S, Yang Z, Chu J (2018) Correlations among Apparent Diffusion Coefficient and Permeability Parameters from Dynamic Contrast-enhanced MR in Brain Tumor Parenchyma and Peritumoral Area. J Neurol Neurophysiol 9: 470. doi:10.4172/2155-9562.1000470

Page 4 of 18

\begin{tabular}{|c|c|c|c|c|c|c|c|c|c|c|c|}
\hline \multirow[b]{2}{*}{ ROls } & \multirow{2}{*}{$\begin{array}{c}\text { Parameters } \\
\text { being correlated } \\
\text { with ADC }\end{array}$} & \multicolumn{2}{|c|}{ LGG } & \multicolumn{2}{|c|}{ HGG } & \multicolumn{2}{|l|}{ PCNSL } & \multicolumn{2}{|c|}{ Metastases } & \multicolumn{2}{|c|}{ Meningiomas } \\
\hline & & $r$ & $\mathrm{p}$ & $r$ & $\mathrm{p}$ & $r$ & $\mathrm{p}$ & $r$ & $\mathrm{p}$ & $r$ & $\mathrm{p}$ \\
\hline \multirow{4}{*}{ TP } & $\mathrm{K}^{\text {trans }}$ & -0.33 & $0.002^{*}$ & -0.24 & $0.004^{*}$ & -0.58 & $<0.000^{*}$ & 0.13 & 0.39 & 0.30 & $0.005^{*}$ \\
\hline & Ve & -0.41 & $<0.000^{\star}$ & -0.29 & $<0.000^{\star}$ & -0.52 & $<0.000^{*}$ & 0.32 & $0.04^{\star}$ & -0.03 & 0.77 \\
\hline & iAUC & -0.37 & $<0.000^{*}$ & -0.34 & $<0.000^{*}$ & -0.60 & $<0.000^{*}$ & 0.23 & 0.14 & 0.32 & $0.003^{\star}$ \\
\hline & Kep & 0.59 & $<0.000^{\star}$ & 0.29 & $<0.000^{*}$ & -0.01 & 0.92 & -0.17 & 0.26 & 0.42 & $<0.000^{*}$ \\
\hline \multirow{4}{*}{ PT } & $\mathrm{K}^{\text {trans }}$ & -0.32 & $0.03^{*}$ & -0.34 & $0.003^{*}$ & -0.12 & 0.55 & -0.25 & 0.23 & -0.15 & 0.29 \\
\hline & $\mathrm{Ve}$ & -0.32 & $0.03^{*}$ & -0.48 & $<0.000^{*}$ & -0.21 & 0.26 & -0.31 & 0.13 & -0.33 & $0.02^{*}$ \\
\hline & iAUC & -0.45 & $0.002^{*}$ & -0.59 & $<0.000^{*}$ & -0.58 & $0.001^{*}$ & -0.33 & 0.11 & -0.50 & $<0.000^{*}$ \\
\hline & Kep & -0.07 & 0.67 & 0.37 & $0.001^{\star}$ & 0.57 & $0.001^{*}$ & 0.28 & 0.18 & 0.28 & $0.049^{*}$ \\
\hline
\end{tabular}

Table 2: Correlations between each of the permeability parameter and ADC in different brain tumors and ROIs (TP: Tumor Parenchyma; PT: Peri Tumoral area; LGG: Low Grade Gliomas; HGG: High Grade Gliomas; PCNSL: Primary Central Nerveous System Lymphoma; *Significant.

HGG

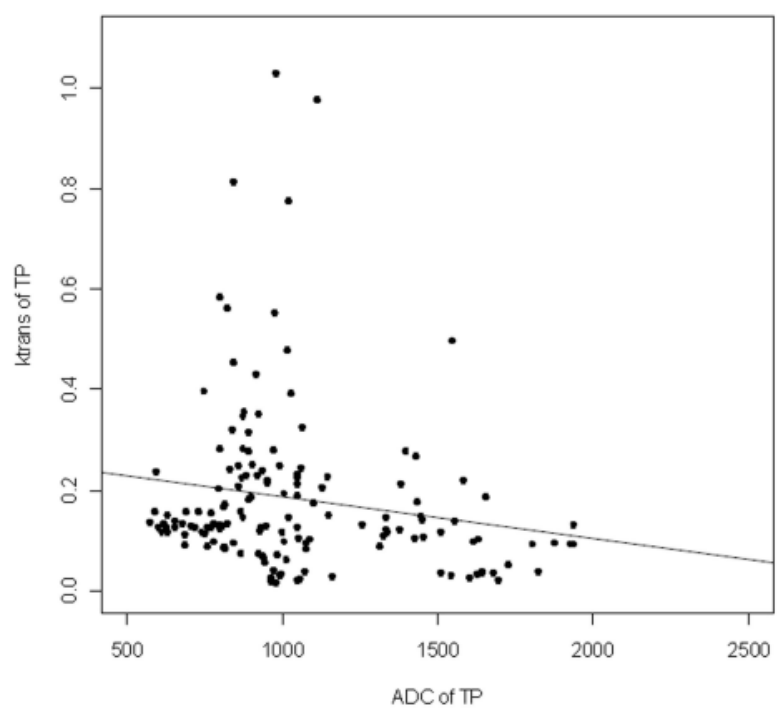

HGG

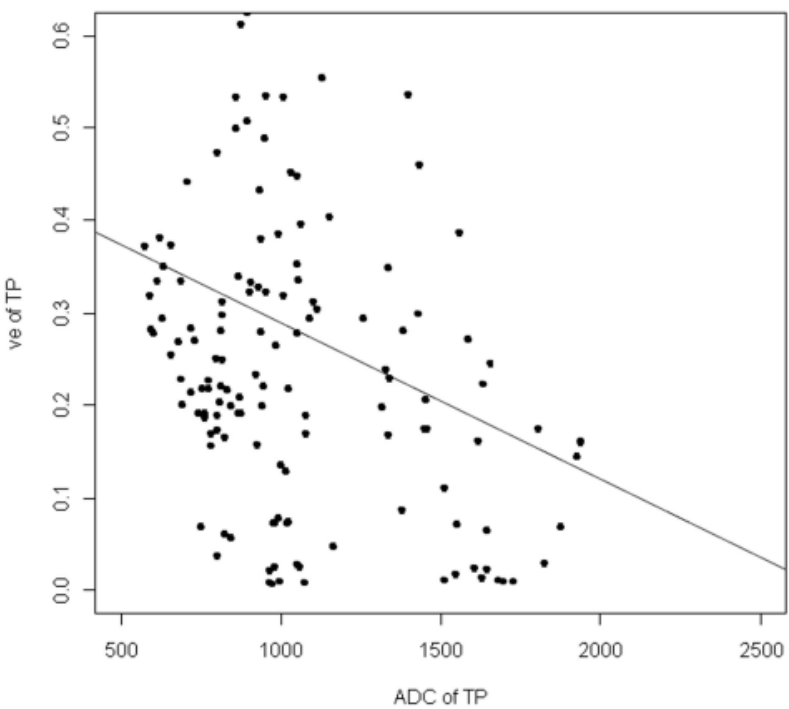

Meningiomas

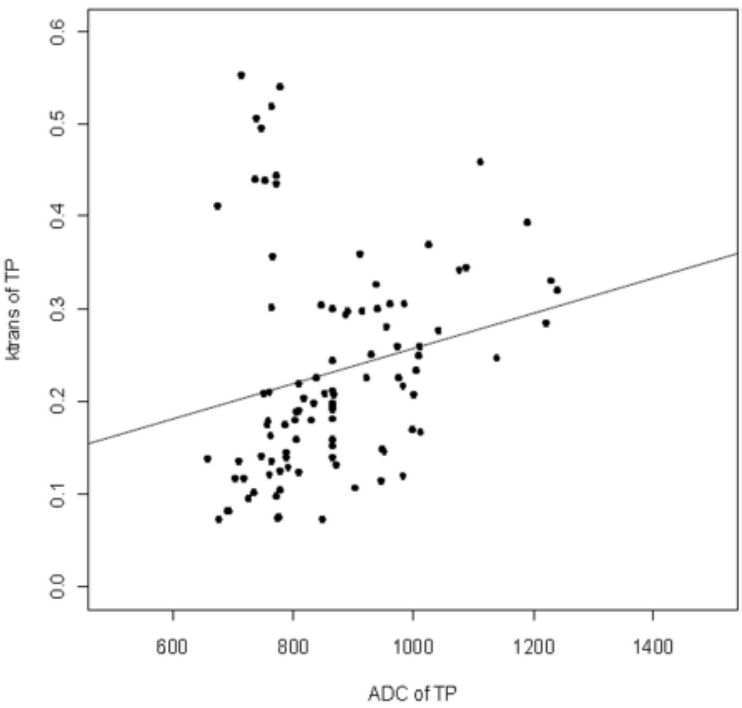

Metastases

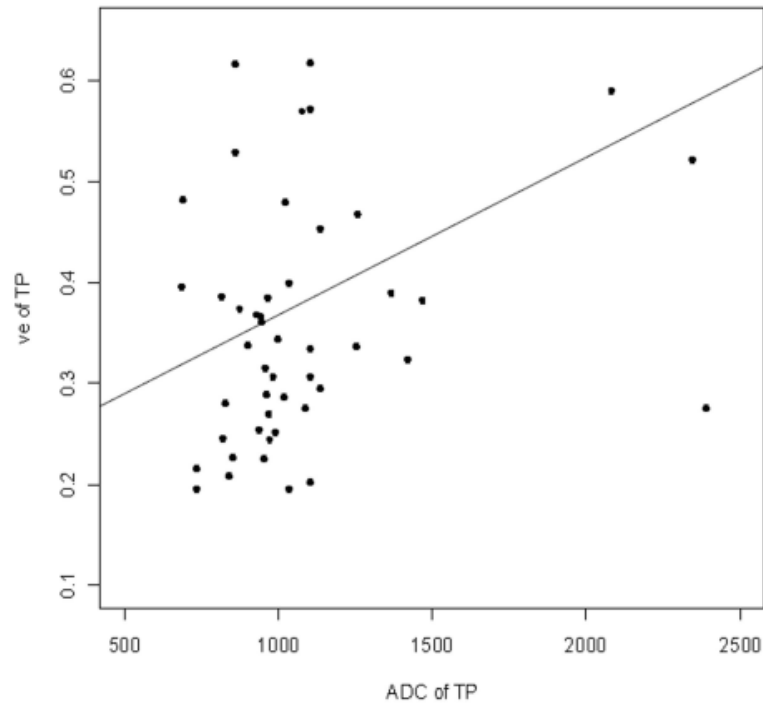

Figure 3: Scatter plot for Ktrans, Ve with ADC in Tumor Parenchyma (TP) of High Grade Gliomas (HGG), meningioma and metastases. 
Citation: Zhao J, Feng Y, He S, Yang Z, Chu J (2018) Correlations among Apparent Diffusion Coefficient and Permeability Parameters from Dynamic Contrast-enhanced MR in Brain Tumor Parenchyma and Peritumoral Area. J Neurol Neurophysiol 9: 470. doi:10.4172/2155-9562.1000470

correlations among permeability parameters in diverse types of brain tumor based on different ROIs were shown in Table 3. Among the permeability parameters, no matter whether the selected ROIs were in TP or PT area, the mean values of Ktrans, Ve and iAUC were mutually significantly positively associated with each other. The HGG, which served as an example were shown in (Figure 4). Taking all the data into consideration, the correlation coefficients were higher in TP than in PT area. Furthermore, with different brain tumor types and different regions of ROIs, the correlations between Kep and other permeability parameters were different (Table 3).

Nowadays, advanced MRI technique, such as DWI and DCE-MR are wildly used to investigate brain tumor micro-environment. The ADC values and permeability parameters (Ktrans, Ve, Kep and iAUC), which are claimed as promising imaging biomarkers that can provide information to reflect tumor cell number, size, packing way and microcirculation. Few studies have already showed that there exist correlations among them in tumors $[20,29]$. In order to better understand their correlations and utilize these parameters in brain tumor differentiation, there is a persistent requirement for performing a more extensive study including multiple perfusion and diffusion parameters in TP and PT area. As reported in literatures, Ve reflected the contrast agent's volume distributed in the interstitial space and the mean value of Ve was

\begin{tabular}{|c|c|c|c|c|c|c|c|c|c|c|c|c|c|}
\hline \multirow[b]{2}{*}{ ROls } & \multirow{2}{*}{$\begin{array}{c}\text { Brain tumor } \\
\text { type }\end{array}$} & \multicolumn{2}{|c|}{ Ktrans with Ve } & \multicolumn{2}{|c|}{ Ktrans with iAUC } & \multicolumn{2}{|c|}{ Ve with iAUC } & \multicolumn{2}{|c|}{ Kep with Ktrans } & \multicolumn{2}{|c|}{ Kep with iAUC } & \multicolumn{2}{|c|}{ Kep with Ve } \\
\hline & & $r$ & $\mathrm{p}$ & $r$ & $\mathrm{p}$ & $r$ & $\mathrm{p}$ & $r$ & $\mathrm{p}$ & $r$ & $\mathrm{p}$ & $r$ & $\mathrm{p}$ \\
\hline \multirow{5}{*}{ TP } & LGG & 0.93 & $<0.000^{\circ}$ & 0.90 & $<0.000^{*}$ & 0.92 & $<0.000^{*}$ & -0.42 & $<0.000^{*}$ & -0.59 & $<0.000^{*}$ & -0.64 & $<0.000^{*}$ \\
\hline & HGG & 0.57 & $<0.000^{*}$ & 0.68 & $<0.000^{*}$ & 0.87 & $<0.000^{\circ}$ & -0.03 & 0.69 & -0.47 & $<0.000^{*}$ & -0.72 & $<0.000^{*}$ \\
\hline & PCNSL & 0.86 & $<0.000^{*}$ & 0.94 & $<0.000^{*}$ & 0.83 & $<0.000^{*}$ & 0.006 & 0.96 & 0.06 & 0.634 & -0.24 & 0.06 \\
\hline & Metastases & 0.47 & $0.001^{*}$ & 0.96 & $<0.000^{*}$ & 0.61 & $<0.000^{*}$ & 0.65 & $<0.000^{*}$ & 0.52 & $<0.000^{*}$ & -0.27 & 0.06 \\
\hline & Meningioma & 0.58 & $<0.000^{\circ}$ & 0.91 & $<0.000^{*}$ & 0.48 & $<0.000^{*}$ & 0.76 & $<0.000^{\circ}$ & 0.74 & $<0.000^{*}$ & -0.06 & 0.56 \\
\hline \multirow{5}{*}{ PT } & LGG & 0.40 & $0.007^{*}$ & 0.32 & $0.03^{*}$ & 0.88 & $<0.000^{*}$ & 0.58 & $<0.000^{*}$ & -0.40 & $0.007^{*}$ & -0.38 & $0.01^{*}$ \\
\hline & HGG & 0.59 & $<0.000^{*}$ & 0.67 & $<0.000^{\circ}$ & 0.93 & $<0.000^{*}$ & 0.10 & 0.41 & -0.59 & $<0.000^{*}$ & -0.65 & $<0.000^{*}$ \\
\hline & PCNSL & 0.51 & $0.004^{*}$ & 0.64 & $<0.000^{*}$ & 0.84 & $<0.000^{*}$ & -0.28 & 0.13 & -0.82 & $<0.000^{*}$ & -0.77 & $<0.000^{*}$ \\
\hline & Metastases & 0.80 & $<0.000^{*}$ & 0.87 & $<0.000^{*}$ & 0.88 & $<0.000^{\circ}$ & -0.65 & $<0.000^{*}$ & -0.86 & $<0.000^{*}$ & -0.71 & $<0.000^{*}$ \\
\hline & Meningiomas & 0.51 & $<0.000^{*}$ & 0.58 & $<0.000^{*}$ & 0.86 & $<0.000^{\circ}$ & -0.01 & 0.97 & -0.67 & $<0.000^{*}$ & -0.62 & $<0.000^{*}$ \\
\hline
\end{tabular}

Table 3: Correlations among permeability parameters in different types of brain tumor which are based on different ROls (TP: Tumor Parenchyma; PT: Peri Tumoral area LGG: Low Grade Gliomas; HGG: High Grade Gliomas; PCNSL: Primary Central Nervous System Lymphoma; *Significant.

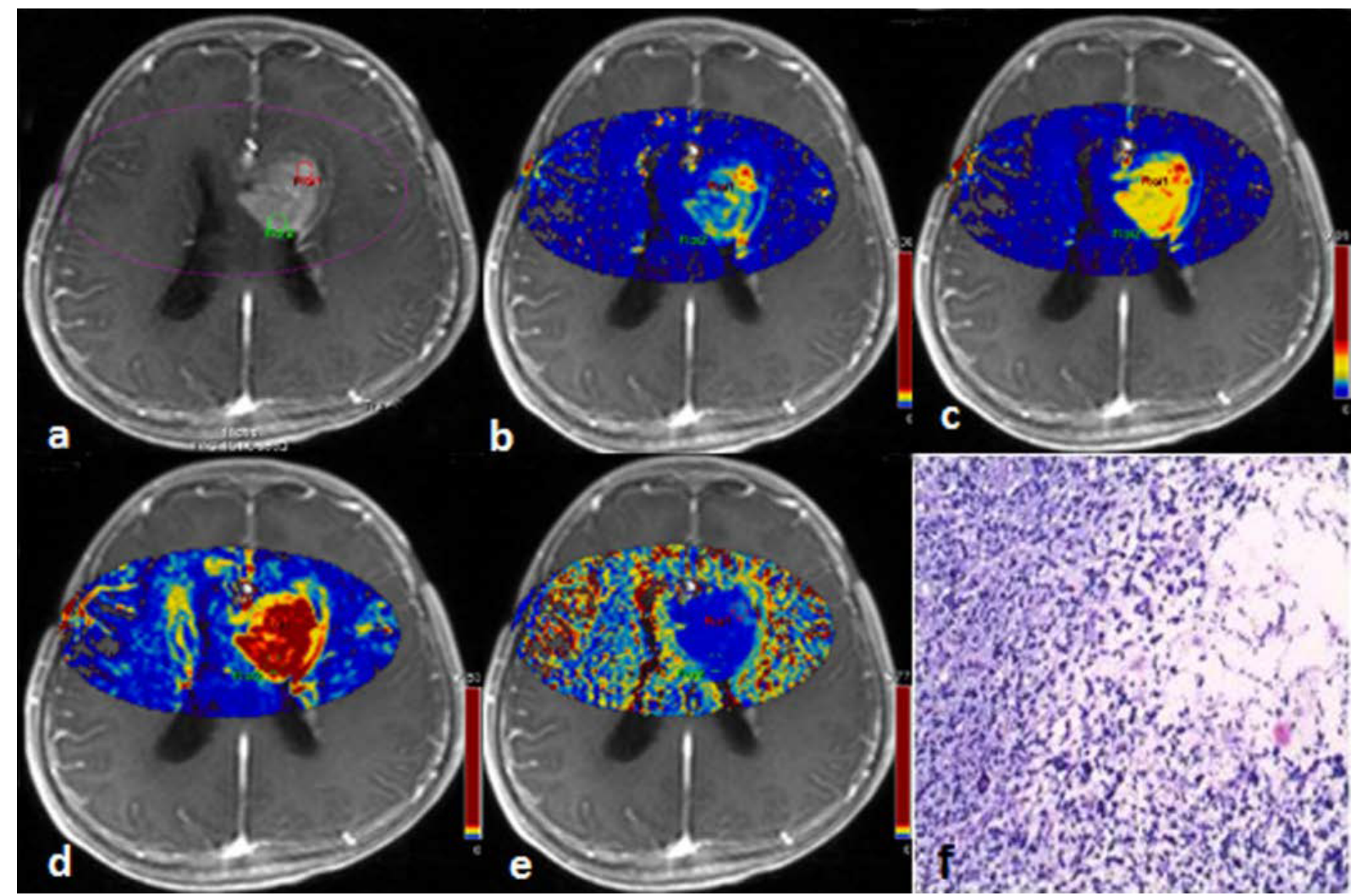

Figure 4: An example of high grade glioma with DCE-MRI examination. A young man with high-grade gliomas (WHO IV) and the tumor affected the left centrum semiovale, corpus callosum body and basal ganglia. Enhanced, Ktrans, Ve, iAUC and Kep maps (a-e) showed that tumor manifested with homogeneous enhancement and the mean values of Ktrans, Ve and iAUC were accordingly higher while the mean value of Kep were lower; histologic results; (f): tissue from tumor parenchyma was proved as glioblastoma multiforme, (Hematoxylin-Eosin $(\mathrm{HE}) \times 10)$. 
correlated with the amount of temporary trapping of the contrast agent in interstitial space, which is dependent on endothelial changes and the interstitial environment [30]. While Ktrans and Kep are related to endothelial changes, Ktrans represent the transfer of contrast agent from the plasma to the interstitial space while Kep is the reverse transfer rate from the EES to the plasma space. Further, iAUC is determined by the amount of the trapped contrast agent in plasma and interstitial space [31]. Hence, tumors with higher Ktrans and lower Kep which means more contrast agent would be trapped in interstitial space could lead to a higher Ve, the higher Ktrans and Ve combined were contributed to the higher iAUC. The interpretation of each permeability parameter could reasonably explain our results that, irrespective of brain tumor types and ROI positions, the mean Ktrans, Ve and iAUC values were mutually strongly positively associated with each other (Table 1 and Figure 4).

Deeply and more specifically, in TP area, Figure 3a demonstrated that iAUC and Ve, iAUC and Ktrans equally showed the highest correlative relationship, and then followed by Ve and Ktrans, which confirmed the hypothesis that the higher Ktrans and Ve combined were contributed to the higher iAUC. Moreover, for Ktrans, the most correlative parameter is iAUC, followed by Ve and Kep. There was no doubt that as the increase ofcapillary permeability, the value of iAUC would increase either. Further, Kep negatively correlated with iAUC $(r T P=-0.36 ; r P T=-0.68)$ and $\mathrm{Ve}(\mathrm{rTP}=-0.64 ; \mathrm{rPT}=-0.67)$, the $\mathrm{r}$ is higher in PT area. Although Ktrans and kep are both related to endothelial changes, we noticed that their $r$ is the lowest. Even there was no significant correlation was found in PT area (Table 1). These might inspire us that the endothelial changes and the size of EES which acted together to influence the Kep value and the Kep value were more depended on the volume of EES. Another interesting finding which we detected was the negative correlation between Kep and Ve. As showed in the equation Kep=Ktrans/Ve, the negative correlation of Kep and Ve might depend on the balance between capillary permeability and blood flow in the tissue of interest [24].

As specified to each type of brain tumor, no matter the ROIs in TP or PT area, the positive correlations among Ktrans, Ve and iAUC were found (Table 3). However, of other permeability parameters with Kep, the correlations were different from tumor types and ROI positions (Table 3 ). This might indicate the mechanism behind Kep was even more complicated and more related with the specific tumor type. Radjenovic et al. [15] found that Kep value was significantly higher in grade three tumors than in low grade tumor and Li et al. [32] reported that the reduction of Kep value was associated with a better response to chemo-therapy. Therefore, Kep might have a higher possibility to be chosen as an imaging biomarker to differentiate brain tumors and monitoring tumor treatment response.

There was a study showed that, irrespective of brain tumor types, mean ADC, in TP area, were significantly inversely correlated with mean Ktrans, Ve and iAUC and positively correlated with mean Kep [20]. A similar result was reported by Langer et al. [29] in prostate cancer. Our study not only shows the comparable results in TP but also in PT area. To our knowledge, these correlations have not been reported in PT area. Our findings suggested that there is an intricate relationship between water diffusion property and capillary membrane permeability in and around tumoral area.

There were several factors could affect permeability parameters and ADC value, such as tumor cellularity, the ischemia degree of tumor cell, capillary bed perfusion, permeability, extracellular matrix composition and interstitial edema $[6,8,20,29]$. Several groups have reported an inverse correlation of ADC with cell density [5-8]. High grade tumors have a higher cellularity and tend to have an increase in immature tumoral vessels, which might induce higher Ktrans. These might explain the inverse correlation between Ktrans and ADC. Yankeelov et al. [33] showed the comparable results in pre-treatment breast cancer. Further, based on our own and previous studies's results (Table 1). The Ktrans, Ve and iAUC were changed coincidentally and dynamically with the growth of a tumor $[21,22,31]$ which might explain the similar correlations of Ktrans, Ve, iAUC with ADC. Kep, relied on our own results, was negatively correlated with Ktrans, Ve and iAUC (Table 1). Thus, we could assume that Kep might positively related with ADC and our results confirmed this assumption. Based on the positive correlation between Kep and ADC, we might reasonably infer that Kep not only related with vessel permeability but also with the tumor cellularity, since ADC values negatively correlate with tumor cellularity.

However, as for $\mathrm{ADC}$ value, in $\mathrm{TP}$, the most negative correlation was Ve, and then followed by iAUC and Ktrans, which, to some extent, could be interpreted as the higher of the tumor cellularity (lower ADC value), the greater of the EES (higher Ve value). Sometime Ve also referred to tumor necrosis $[13,22]$ and the tumor cells were not tightly packaged if tumor necrosis occurred. The different correlations among permeability parameters and ADC in TP and PT area within a specific brain tumor might be explained by the different histologic features (location, growth manner and the vessel supply) of each type of brain tumor [34] (Table 2). Interestingly, we noticed that the correlations of permeability parameters with ADC in gliomas and PCNSL are changed in an opposite way with the correlations of permeability parameters and ADC in meningioma and metastases (Figure 3). The probable reason is that glioma and lymphoma that are inherent to brain parenchyma have Blood Brain Barrier (BBB), even though they would destroy BBB. However, metastasis or meningioma does not have $\mathrm{BBB}$.

Therefore, based on our findings, we possibly infer that: 1) Ktrans, Ve and iAUC may have comparable diagnostic power for discrimination of brain tumors since they are mutually positively correlated with each other; 2) Kep may not only relate with vessel permeability but also with the tumor cellularity and the size of EES, probably more depend on the size of EES; 3) ADC and Ve, in TP, showed the most negative correlation, which, to some extent, could be interpreted as the higher of the tumor cellularity and the greater of the EES. However, the relatively simple permeation model (Tofts model), ROI-based method, and various brain tumors included, the exact mechanisms behind these correlations were sophisticated and a follow up study in this direction should be carried on. We expect our results to contribute to perfusion analyses in diverse of clinical and research settings.

\section{Conclusion}

In conclusion, Intricate correlations were found among permeability parameters and ADC, irrespective of the brain tumor types and ROI positions. Ktrans, Ve and iAUC may have comparable diagnostic power for discrimination of brain tumors. Kep may not only relate with vessel permeability but also with the tumor cellularity and the size of EES, probably more depend on the size of EES and ADC with Ve. In TP, it showed the most negative correlation, which, to some extent, could be interpreted as the higher the tumor cellularity and the greater the EES. These findings would help in better understanding of those permeability parameters and ADC.

\section{References}

1. Ostrom QT, Gittleman H, Farah P, Ondracek A, Chen Y, et al. (2013) CBTRUS statistical report: Primary brain and central nervous system tumors diagnosed in the United States in 2006-2010. Neuro Oncol 15: 1-56. 
Citation: Zhao J, Feng Y, He S, Yang Z, Chu J (2018) Correlations among Apparent Diffusion Coefficient and Permeability Parameters from Dynamic Contrast-enhanced MR in Brain Tumor Parenchyma and Peritumoral Area. J Neurol Neurophysiol 9: 470. doi:10.4172/2155-9562.1000470

Page 7 of 18

2. Burger P (1990) Classification, grading and patterns of spread of malignant gliomas neurosurgical topics: Malignant cerebral glioma. Park Ridge, III. American Association of Neurological Surgeons, pp: 3-17.

3. Lee EJ, Ahn KJ, Lee EK, Lee YS, Kim DB (2013) Potential role of advanced MRI techniques for the peritumoural region in differentiating glioblastoma multiforme and solitary metastatic lesions. Clin Radiol 68: e689-e697.

4. Svolos P, Tsolaki E, Kapsalaki E, Theodorou K, Fountas K, et al. (2013) Investigating brain tumor differentiation with diffusion and perfusion metrics at 3T MRI using pattern recognition techniques. Magn Reson Imaging 31: 15671577.

5. Chenevert TL, Stegman LD, Taylor JM, Robertson PL, Greenberg HS, et al (2000) Diffusion magnetic resonance imaging: An early surrogate marker of therapeutic efficacy in brain tumors. J Natl Cancer Inst 92: 2029-2036.

6. Sugahara T, Korogi Y, Kochi M, Ikushima I, Shigematu Y, et al. (1999) Usefulness of diffusion-weighted MRI with echo-planar technique in the evaluation of cellularity in gliomas. J Magn Reson Imaging 9: 53-60.

7. Kono K, Inoue Y, Nakayama K, Shakudo M, Morino M, et al. (2001) The role of diffusion-weighted imaging in patients with brain tumors. AJNR Am J Neuroradiol 22: 1081-1088.

8. Guo AC, Cummings TJ, Dash RC, Provenzale JM (2002) Lymphomas and high-grade astrocytomas: Comparison of water diffusibility and histologic characteristics. Radiol 224: 177-183.

9. Sen PN, Basser PJ (2005) A model for diffusion in white matter in the brain Biophys J 89: 2927-2938.

10. Lacerda S, Law M (2009) Magnetic resonance perfusion and permeability imaging in brain tumors. Neuroimaging Clin N Am 19:527-557.

11. Thompson G, Mills SJ, Stivaros SM, Jackson A (2010) Imaging of brain tumors: perfusion/permeability. Neuroimaging Clin N Am 20: 337-353.

12. Cha S, Yang L, Johnson G, Lai A, Chen MH, et al. (2006) Comparison of microvascular permeability measurements, $\mathrm{K}($ trans), determined with conventional steady-state T1-weighted and first-pass T2*-weighted MR imaging methods in gliomas and meningiomas. AJNR Am J Neuroradiol 27:409-417.

13. Mills SJ, Soh C, Rose CJ, Cheung S, Zhao S, et al. (2010) Candidate biomarkers of extravascular extracellular space: A direct comparison of apparent diffusion coefficient and dynamic contrast-enhanced MR imaging derived measurement of the volume of the extravascular extracellular space in glioblastoma multiforme. AJNR Am J Neuroradiol 31:549- 553

14. Koo HR, Cho N, Song IC, Kim H, Chang JM, et al. (2012) Correlation of perfusion parameters on dynamic contrast-enhanced MRI with prognostic factors and subtypes of breast cancers. J Magn Reson Imaging 36: 145-151.

15. Radjenovic A, Dall BJ, Ridgway JP, Smith MA (2008) Measurement of pharmacokinetic parameters in histologically graded invasive breast tumours using dynamic contrast-enhanced MRI. Br J Radiol 81:120-128.

16. Evelhoch JL (1999) Key factors in the acquisition of contrast kinetic data for oncology. J Magn Reson Imaging 10:254-9.

17. Jia Z, Geng D, Xie T, Zhang J, Liu Y (2012) Quantitative analysis of neovascular permeability in glioma by dynamic contrast-enhanced MR imaging. J Clin Neurosci 19: 820-823.

18. Awasthi R, Rathore RK, Soni P, Sahoo P, Awasthi A, et al. (2012) Discriminant analysis to classify glioma grading using dynamic contrast-enhanced MRI and immunohistochemical markers. Neuroradiol 54: 205-213.
19. Zhao J, Yang ZY, Luo BN, Yang JY, Chu JP (2015) Quantitative evaluation of diffusion and dynamic contrast-enhanced MR in tumor parenchyma and peritumoral area for distinction of brain tumors. PLoS One 10: e0138573.

20. Chu JP, Mak HK, Yau KK, Zhang L, Tsang J, et al. (2012) Pilot study on evaluation of any correlation between MR perfusion (Ktrans) and diffusion (apparent diffusion coefficient) parameters in brain tumors at 3 Tesla. Cancer Imaging 12: 1-6.

21. Mills SJ, Soh C, O'Connor JP, Rose CJ, Buonaccorsi G, et al. (2010) Enhancing fraction in glioma and its relationship to the tumoral vascular microenvironment: a dynamic contrast-enhanced MR imaging study. AJNR Am J Neuroradiol 31 : 726-731.

22. Choi HS, Kim AH, Ahn SS, Shin NY, Kim J, et al. (2013) Glioma grading capability: Comparisons among parameters from dynamic contrast-enhanced MRI and ADC value on DWI. Korean J Radiol 14: 487-492.

23. Di Chiro G (1989) Meningioma subtypes: MR and PET features. Radiol 172 578-589.

24. Tofts PS, Brix G, Buckley DL, Evelhoch JL, Henderson E, et al. (1999) Estimating kinetic parameters from dynamic contrast-enhanced T(1)-weighted MRI of a diffusible tracer: Standardized quantities and symbols. J Magn Reson Imaging 10: 223-232.

25. Tofts PS (1997) Modeling tracer kinetics in dynamic Gd-DTPA MR imaging. J Magn Reson Imaging 7: 91-101.

26. Law M, Cha S, Knopp EA, Johnson G, Arnett J, et al. (2002) High-grade gliomas and solitary metastases: Differentiation by using perfusion and proton spectroscopic MR imaging. Radiol 222: 715-721.

27. Weber MA, Thilmann C, Lichy MP, Günther M, Delorme S, et al. (2004) Assessment of irradiated brain metastases by means of arterial spin-labeling and dynamic susceptibility-weighted contrast-enhanced perfusion MRI: initial results. Invest Radiol 39: 277-87.

28. Weber MA, Zoubaa S, Schlieter M, Jüttler E, Huttner HB, et al. (2006) Diagnostic performance of spectroscopic and perfusion MRI for distinction of brain tumors. Neurol 66: 1899-1906.

29. Langer DL, van der Kwast TH, Evans AJ, Plotkin A, Trachtenberg J, et al (2010) Prostate tissue composition and MR measurements: Investigating the relationships between $A D C, T 2, K($ trans), $\mathrm{v}(\mathrm{e})$, and corresponding histologic features. Radiol 255: 485-494.

30. Knopp MV, von Tengg-Kobligk H, Choyke PL (2003) Functional magnetic resonance imaging in oncology for diagnosis and therapy monitoring. Mo Cancer Ther 2: 419-426.

31. Cuenod CA, Balvay D (2013) Perfusion and vascular permeability: Basic concepts and measurement in DCE-CT and DCE-MRI. Diagn Interv Imaging 94: 1187-1204.

32. Li X, Arlinghaus LR, Ayers GD, Chakravarthy AB, Abramson RG, et al. (2014) DCE-MRI analysis methods for predicting the response of breast cancer to neoadjuvant chemotherapy: pilot study findings. Magn Reson Med 71: 15921602.

33. Yankeelov TE, Lepage M, Chakravarthy A, Broome EE, Niermann KJ, et al (2007) Integration of quantitative DCE-MRI and ADC mapping to monitor treatment response in human breast cancer: initial results. Magn Reson Imaging 25: 1-13.

34. Machein MR, Plate KH (2000) VEGF in brain tumors. J Neurooncol 50:109-120. 